\title{
Using patient biomarker time series to determine mortality risk in hospitalised COVID-19 patients: a comparative analysis across two New York hospitals
}

\author{
Ben Lambert ${ }^{1{ }^{*}}$, Isaac J. Stopard ${ }^{2 \diamond}$, Amir Momeni-Boroujeni ${ }^{3}$, Rachelle Mendoza ${ }^{4}$, \\ Alejandro Zuretti ${ }^{5}$, \\ 1 Department of Computer Science, University of Oxford, Oxford, Oxfordshire, UK \\ 2 MRC Centre for Global Infectious Disease Analysis, School of Public Health, Faculty \\ of Medicine, Imperial College London, London UK \\ 3 Department of Pathology, Memorial Sloan Kettering Cancer Center, New York, NY, \\ USA
}

4 SUNY Downstate Health Sciences University Department of Pathology, Brooklyn, NY 5 SUNY Downstate Health Sciences University and Maimonides Medical Center, Department of Pathology, Brooklyn, NY 11219

OThese authors contributed equally to this work.

*ben.c.lambert@gmail.com

\section{Abstract}

A large range of prognostic models for determining the risk of COVID-19 patient mortality exist, but these typically restrict the set of biomarkers considered to measurements available at patient admission. Additionally, many of these models are trained and tested on patient cohorts from a single hospital, raising questions about the generalisability of results. We used a Bayesian Markov model to analyse time series data of biomarker measurements taken throughout the duration of a COVID-19 patient's hospitalisation for $n=1540$ patients from two hospitals in New York: State University of New York (SUNY) Downstate Health Sciences University and Maimonides Medical Center. In doing so, we quantified the mortality risk associated with both static (e.g. demographic and patient history variables) and dynamic factors (e.g. changes in biomarkers) throughout hospitalisation. By using our model to make predictions across the hospitals, we assessed how predictive factors generalised between the two cohorts. The individual dynamics of the measurements and their associated mortality risk were remarkably consistent across the hospitals. The model accuracy in predicting patient outcome (death or discharge) was $72.3 \%$ (predicting SUNY; posterior median accuracy) and $71.4 \%$ (predicting Maimonides) respectively. Model sensitivity was higher for detecting patients who would go on to be discharged $(79.2 \%)$ versus those who died (61.0\%). Our results indicate the utility of including dynamic clinical measurements when assessing patient mortality risk but also highlight the difficulty of identifying high risk patients.

\section{Introduction}

As the coronavirus disease 2019 (COVID-19) pandemic continues to overwhelm many health services, accurate prognosis remains essential to improved clinical care and decisions regarding the equitable allocation of insufficient intensive care resources [1]. 
Since the beginning of the pandemic, many novel prognostic factors have been identified and applied in prognostic models to predict the course of infection of hospitalised COVID-19 patients 2. Substantial inequality in the burden of COVID-19 exists, and many social determinants of the outcome of infection have been identified, such as deprivation 3 7]. External validation of novel COVID-19 prognostic factors across a range of different settings is therefore vital. Multivariable models may partially reconcile differences in samples used for model training, though external validation is essential because overfitting and confounding of unknown, yet important, variables are likely to limit the out-of-sample predictive accuracy 8 . Indeed, a systematic validation of 22 prognostic models to an external dataset found none performed better than using the best univariable predictor: age 9]. What's more, of 107 surveyed prognostic models, many were found to suffer from small sample sizes and have a high risk of bias in the dataset participants [2].

A number of biomarkers at presentation, including C-reactive protein, lymphocyte count, oxygen saturation and urea concentration, are important predictors of hospitalised COVID-19 patient deterioration (defined as the requirement of ventilatory support, critical care or death) and were included in a recently developed prognostic model of patient deterioration which achieved robust predictive accuracy (C-statistic: 0.77 ) when internally and externally validated on a dataset of 66,705 patients 10. Similarly, peripheral oxygen saturation, urea level and C-reactive protein at presentation are used to predict patient mortality [11. Patients are, however, admitted to hospital at different states of disease progression, and their biomarkers change throughout the course of hospitalisation 12 15. Emerging evidence indicates a number of time-dependent biomarkers changes may therefore be useful prognostic factors: increases in platelets and eosinophil percentage are indicative of reduced mortality risk, whilst increases in alkaline phosphatase may indicate increased mortality risk [15]. Incorporating dynamic changes in biomarkers can improve the predictive accuracy of prognostic models when internally validated 14,15$]$, but the external validation of these prognostic factors is still required. The role of time-dependent biomarkers in different patients remains a key question [16. We previously developed a prognostic Markov model, which allows the quantification of daily mortality risk and the impact of dynamic changes in biomarkers on this quantity and fit the model to data from State University of New York (SUNY) Downstate Medical Center [15. In this study, we fit the model to new data from a different New York hospital: Maimonides Medical Center (henceforth "Maimonides"). We then compare the impact of dynamic changes in patient biomarkers on in-hospital mortality risk (i.e. patient outcomes), across SUNY and Maimonides. In doing so, we obtain an external validation of the model and, more generally, allows us to appraise the use of dynamic biomarker measurements for determining patient mortality risk.

\section{Methods}

\section{Case selection, data extraction and processing}

Study approval was obtained from the State University of New York (SUNY) Downstate Health Sciences University Institutional Review Board (IRB\#1595271-1) and Maimonides Medical Center Institutional Review Board/Research Committee (IRB\#2020-05-07).

A retrospective query was performed among the patients who were admitted to SUNY Downstate Medical Center and Maimonides Medical Center with COVID-19-related symptoms, which was subsequently confirmed by RT PCR, from the beginning of February 2020 until the end of May 2020. Stratified randomization was 
used to select at least 500 patients who were discharged and 500 patients who died due to the complications of COVID-19. Patient outcome was recorded as a binary choice of "discharged" versus "COVID-19 related mortality". Patients whose outcome was unknown were excluded. Demographic, clinical history and laboratory data was extracted from the hospital's electronic health records. The raw data were cleaned and processed for analysis as described in $\S \mathrm{S} 1.2$.

\section{Estimating risk of mortality for variables available at presentation}

To compare the factors affecting mortality risk across the two hospitals, we calculated the odds ratios (ORs) for each of the variables available at presentation. To do so, we converted the initial biomarker values to binary categories: above (1) or below (0) the pooled sample mean across the two hospitals (in our Markov model, discussed later in Methods, we allow continuous, opposed to binarised impacts of variables on patient outcomes). Laboratory tests values at presentation were included only if 150 or more patients in each of the hospital had data for this test available. If laboratory tests were repeated on the first day of admission, we took the mean value taken on this day to be the value at presentation. Odds ratios were calculated for each variable by estimating the proportion dying for each subgroup and then taking the ratio. We assumed the observed counts of individuals expiring were binomially distributed,

$$
X_{0}^{j} \sim \mathcal{B}\left(N_{0}^{j}, \theta_{0}^{j}\right), \quad X_{1}^{j} \sim \mathcal{B}\left(N_{1}^{j}, \theta_{1}^{j}\right),
$$

where $j$ indicates the binary variable (for example, whether an individual was aged 0-40 or whether they had a history of asthma); $X_{0}^{j}$ and $X_{1}^{j}$ indicate the counts of individuals dying for the $j=0$ and $j=1$ subgroups; $N_{0}^{j}$ and $N_{1}^{j}$ are the observed counts of individuals in the $j=0$ and $j=1$ subgroups; and $\theta_{0}^{j}$ and $\theta_{1}^{j}$ are the estimated proportions dying in the corresponding subgroups. Parameters were estimated using a Bayesian framework: the estimated ratio of $\theta_{1}^{j} / \theta_{0}^{j}$ defined the OR for variable $j$ and was estimated by taking 100,000 independent draws from the posterior distributions of each of $\theta_{1}^{j}$ and $\theta_{0}^{j}$, assuming uniform priors.

\section{Laboratory value time trends}

To determine average trends in laboratory values over the course of a patient's hospitalisation, we carried out a series of regressions for each laboratory test stratified by patient outcome and hospital. To do so, we calculated the percentage change in each patients' biomarker values relative to their values at presentation. These were scaled to have a mean of 0 and standard deviation of 1 . Infinite (i.e. when the first biomarker value was zero), missing or extreme observations (the absolute value of the percentage change exceeded the $98 \%$ quantile) were excluded from the regression so that our results focused on the bulk of observations, opposed to the extremes. The percentage change in test value was modelled as a function of a quadratic time trend, allowing for fixed effect trends but including individual patient slopes of both the linear and quadratic terms of the trend. These models were estimated in a frequentist framework using the lme $4 \mathrm{R}$ package [17, and we extracted the fixed effect estimates of the trends for each model.

\section{Bayesian Markov models of dynamic risk}

The univariate OR estimates described thus far do not account for the impact of other covariates when determining risk. Furthermore, these methods consider a static outcome (whether a patient dies at some point during their hospitalisation), do not account for 
the time taken for the outcome to occur, or allow dynamic variables to be included. A patient's underlying risk of death may, however, change throughout the course of their hospitalisation, which can be indicated by changes in certain biomarkers 15 .

Here, we briefly describe a multivariate discrete time Markov model which aims to identify the importance of different prognostic factors on COVID-19 mortality risk and estimate the change in individual patients' mortality risk throughout the course of hospitalisation. (The model has previously been described more fully here: [15.) The model specifically accounts for the competing risks of discharge and death. Note, it is possible to use cause-specific hazards models (Cox regressions for each event of interest, treating the other event as censored) to estimate the cumulative incidence function but, in these models, it is not possible to assess the impact of individual covariates on the cumulative incidence function 18, 19]. By considering the sequence of outcomes for each day each patient was in hospital, Markov models can simultaneously account for the risk of discharge versus mortality: on the first day, patients are admitted and begin in the "hospital" state; at the end of the first day, they either remain in hospital or transition to the "discharged" or "death" states. On subsequent days, patients that remained in hospital can complete the same possible transitions. The probabilities different transitions occur were modelled as a function of each patient's demographic characteristics, comorbidities, laboratory test values at presentation and dynamic trends in laboratory test values (as measured by their percentage changes relative to their values at presentation). A schematic of the model is provided in Fig 1. The un-normalised probabilities of each possible transition are modelled using a log link:

$$
q_{i t}^{\text {discharged }}=\exp \left(\alpha_{0 i}+\boldsymbol{\alpha}_{1}^{\prime} \boldsymbol{x}_{i t}\right), \quad q_{i t}^{\text {death }}=\exp \left(\beta_{0 i}+\boldsymbol{\beta}_{1}^{\prime} \boldsymbol{x}_{i t}\right), \quad q_{i t}^{\text {hospital }}=1,
$$

where $i$ indicates a given patient; $t$ indicates the day of hospital stay post-admission for a given patient; $\boldsymbol{\alpha}_{1}$ and $\boldsymbol{\beta}_{1}$ are vectors of regression coefficients relating to the vector of (potentially time-varying) regressors in $\boldsymbol{x}_{i t} ; \alpha_{0 i}$ and $\beta_{0 i}$ are patient-specific intercepts. The normalised probabilities of transitions between the states are then given by the ratio of the un-normalised probabilities to the sum of all these:

$q_{i t}^{\text {total }}=q_{i t}^{\text {discharged }}+q_{i t}^{\text {death }}+q_{i t}^{\text {hospital }} ;$ so that, for example, $p_{i t}^{\text {discharged }}=q_{i t}^{\text {discharged }} / q_{i t}^{\text {total }}$.

$$
\text { admitted day } 1 \quad \text { day } 2 \text { day } 3 \text { day } 4
$$

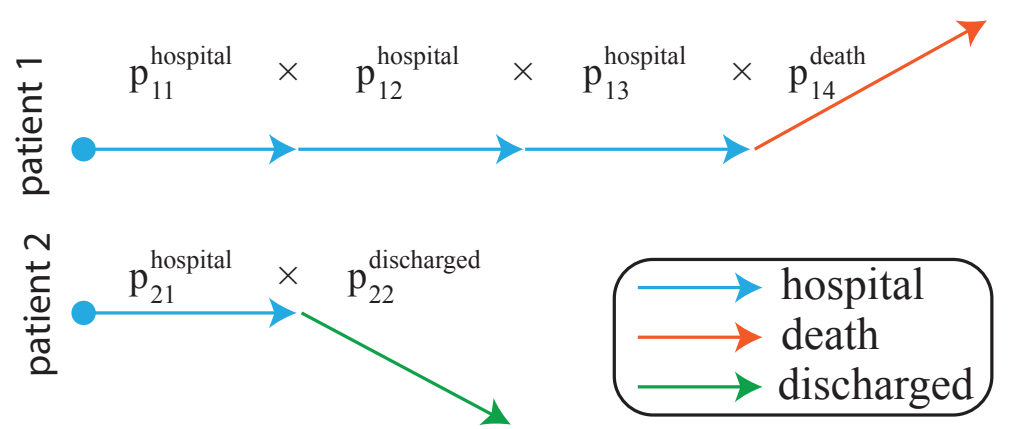

Fig 1. Markov model of patient trajectories during clinical care. For two hypothetical patients, we illustrate how the probability of their observed trajectory is calculated. Note that $p_{i t}$ refers to the probability of an observed transition, which is a function of the patient $(i)$ and day $(t)$ : the time-dependence of probabilities is realised through eq. (2) and is due to (potential) changes in covariates.

Using this model, we performed five separate regressions, each with different groups of independent variables (i.e. different $\boldsymbol{x}_{i t}$ in eq. (2)). In the first of these, we included 
only a single variable in an analysis to examine the influence of each variable in isolation. The second regression included patient demographic characteristics ("patient" variables), including their age, sex and ethnicity, and the day they were admitted to hospital. The third regression ("pat. + comorbidities") supplemented the patient variables with the recorded comorbidities for each patient: whether they had hypertension, diabetes etc. ( $n=13$ conditions in total). The fourth regression ("admission") supplemented the third with the initial measurements for each patient for each of the $n=19$ clinical tests common across the two hospitals. The final regression ("post-admission") then included the percentage changes in each clinical test measurement from the initial values for each patient. Both the initial values and the dynamic values were scaled to give a mean of 0 and a standard deviation of 1 , so that the ORs were estimated on a scale that was consistent across the different laboratory tests and represented the typical clinical variation in these values. The biomarkers included in the study along with the acronyms used are given in Table S1.

The model was estimated in a Bayesian framework using the Stan's NUTS sampler 20 21. We used priors for the regression coefficients that induce sparsity: meaning that only the most predictive covariates would be estimated to have non-zero effects. The priors for the parameters are shown in Table S2. The univariate models were run for 2000 iterations; the multivariate models were run for either 2000 iterations, then a further 2000-6000 iterations if not converged. In all cases, we ran the model using four chains with the first half of iterations discarded as warm-up. The Markov chains satisfied $\hat{R}<1.01$ and bulk- and tail-ESS $>400$ for all parameters, consistent with convergence. The Stan code for the model is provided in $\S S 1.3$.

To check that the Markov model provided a reasonable fit of the underlying data, we performed a series of posterior predictive checks (see, for example, 22, 23]). For the Markov model with the post-admission set of variables, we compared the model-estimated and actual mortality rates, separately for models trained on data from SUNY and Maimonides. In Figs. S1 \& S2, we show the estimated (black point-ranges) and estimated (orange points) mortalities across groupings of our binary predictor variables: indicating a good correspondence in the majority of cases. In Figs. S3 \& S4, we show a similar plot but for the dynamic biomarkers where we compare mortality rates for groups of individuals with last recorded laboratory values above or below the mean.

\section{Generalisation of predictions}

Next, we assessed whether the sets of factors considered in this paper can be used to predict patient outcomes that generalise across both hospitals. To do so, we fitted the Markov model to data from each hospital in turn, then used it to predict patient outcomes in the other held-out hospital. As discussed in Methods, we scaled both the initial laboratory values and the dynamic values using the sample mean and standard deviation. When performing between-hospital prediction, we used the mean and standard deviation of values of the training hospital to scale variables in the independent hospital test set. This ensured that we only used information available in the training set when making predictions.

\section{Results}

\section{Patient characteristics at admission across the hospitals}

The hospital cohorts ( $n=553$ patients in SUNY; $n=987$ in Maimonides) differed in demographic variables and underlying comorbidities (Table 1). Patients of SUNY 


\begin{tabular}{lll}
\hline Variable & SUNY & Maimonides \\
\hline outcome: discharged & $342(61.8 \%)$ & $496(50.3 \%)$ \\
outcome: expired & $211(38.2 \%)$ & $491(49.7 \%)$ \\
sex: female & $271(50.3 \%)$ & $437(44.3 \%)$ \\
sex: male & $268(49.7 \%)$ & $550(55.7 \%)$ \\
ethnicity: black & $472(86.8 \%)$ & $119(12.3 \%)$ \\
ethnicity: hispanic & $17(3.1 \%)$ & $2(0.2 \%)$ \\
ethnicity: other or unrecorded & $39(7.1 \%)$ & $271(27.5 \%)$ \\
ethnicity: white & $25(4.6 \%)$ & $595(61.7 \%)$ \\
age: $0-40$ & $26(4.8 \%)$ & $116(11.8 \%)$ \\
age: $40-50$ & $43(7.9 \%)$ & $57(5.8 \%)$ \\
age: $50-60$ & $93(17.2 \%)$ & $112(11.3 \%)$ \\
age: $60-70$ & $140(25.8 \%)$ & $201(20.4 \%)$ \\
age: $70-80$ & $137(25.3 \%)$ & $210(21.3 \%)$ \\
age: $80+$ & $103(19.0 \%)$ & $291(29.5 \%)$ \\
\hline asthma & $24(4.4 \%)$ & $89(9.0 \%)$ \\
cancer & $16(2.9 \%)$ & $89(9.0 \%)$ \\
cerebrovascular disease & $25(4.6 \%)$ & $80(8.1 \%)$ \\
congestive heart failure & $23(4.2 \%)$ & $292(29.6 \%)$ \\
chronic kidney disease & $19(3.5 \%)$ & $76(7.7 \%)$ \\
copd & $25(4.6 \%)$ & $96(9.7 \%)$ \\
coronary artery disease & $44(8.0 \%)$ & $391(39.6 \%)$ \\
dementia & $13(2.4 \%)$ & $120(12.2 \%)$ \\
diabetes & $229(41.9 \%)$ & $348(35.3 \%)$ \\
endstage renal disease & $54(9.9 \%)$ & $50(5.1 \%)$ \\
hepatitis & $4(0.7 \%)$ & $24(2.4 \%)$ \\
hyperlipidemia & $103(18.8 \%)$ & $270(27.4 \%)$ \\
hypertension & $350(64.0 \%)$ & $516(52.3 \%)$ \\
\hline
\end{tabular}

Table 1. Summary characteristics of patient groups from the two hospitals. Note, that in some cases, data were missing meaning that patients counts across all shown categories do not aggregate to $n=553$ for SUNY and $n=987$ for Maimonides.

predominantly self-reported as black, whereas those of Maimonides predominantly self-reported as white. Diabetes was more prevalent in the SUNY cohort, whereas coronary artery disease and congestive heart failure were more prevalent in the Maimonides cohort. The presence of multiple conditions within individual patients differed substantially between the two cohorts (Fig S5). There were minor differences in the distributions of the laboratory test values at admission (available across both hospitals) of the two hospital cohorts, with the exception of BASO PCT and MCHC (Fig S6).

\section{Consistent univariate mortality risk for factors available at presentation across both hospitals}

To compare the mortality risk for those variables available at presentation (including demographic variables, comorbidities and laboratory test values at admission), which were common across the two hospitals, we estimated the ORs measuring the risk of death associated with each of the variables.

There was a significant positive correlation between the OR estimates of the demographic and comorbidity variables of the two hospitals (using median posterior 

for Maimonides on those from SUNY: $\hat{\beta}=0.65, t_{19}=3.48, p<0.01$ against $H_{0}: \beta=1$ ). Similarly, there was a significant positive correlation between the OR estimates of the laboratory values at admission between the two hospitals $\left(\rho=0.85, t_{17}=6.77, p<0.01\right)$, and the regression slope was not significantly different from $1\left(t_{17}=0.22, p>0.05\right)$ indicating there was no systematic differences in ORs between hospitals for these variables (Fig $2 \mathrm{~B}$ ).

A.

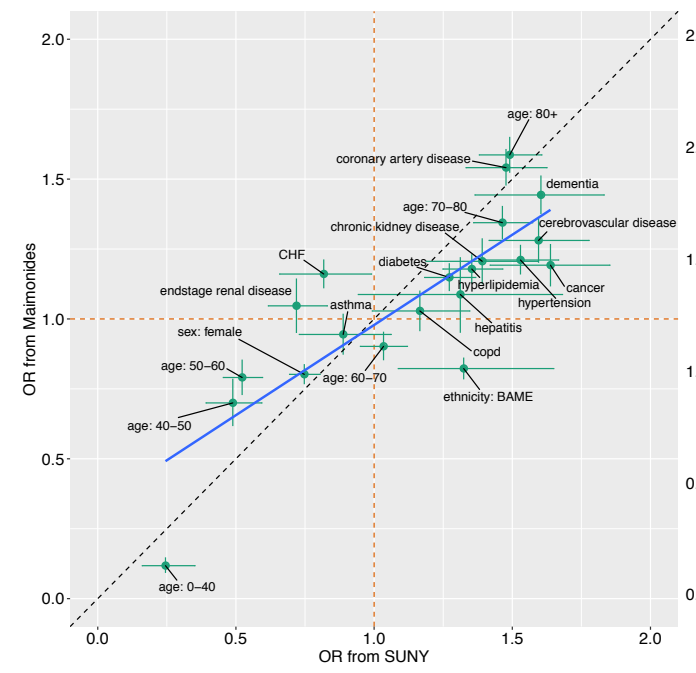

B.

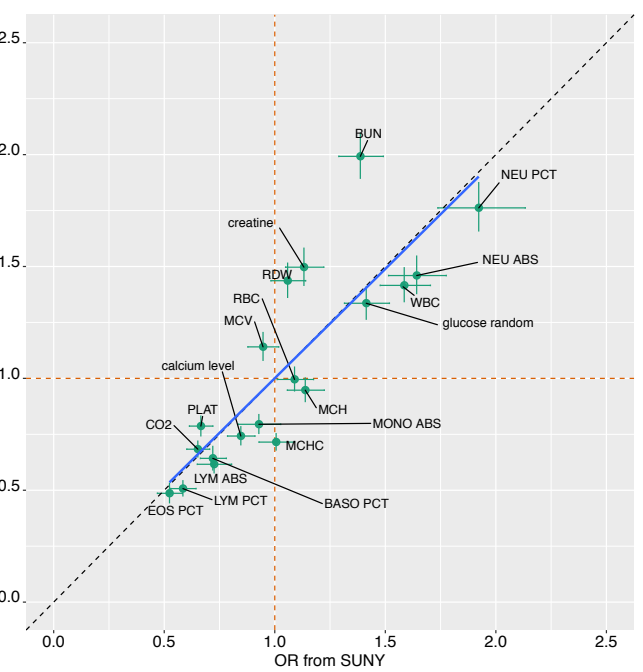

Fig 2. Comparing univariate mortality ORs across the hospitals. The two panels compare ORs associated with demographic and disease history variables (panel A) and initial laboratory test values (panel B). Points show the posterior median ORs; the whiskers display the $25 \%$ and $75 \%$ posterior quantiles. The orange dashed lines show the OR $=1$ cases; the dashed black lines indicate equality in the ORs across the two hospitals. The blue line shows least squares regression lines using the posterior median ORs.

\section{Dynamics of laboratory values}

We next considered dynamic changes in the $n=19$ laboratory biomarkers which were available across both hospitals, which we plot in Fig 3 . This illustrates that, irrespective of patient outcome, there is considerable inter-patient variability in the time series of these biomarkers.

To compare the average dynamics of laboratory test values for patients throughout the course of their hospitalisation, we also estimated hospital-specific time trends for each patient group (see Methods). Across the majority of variables, there was a high degree of correspondence in these average trends across the two hospitals (Fig 3). Indeed, the correlation between the regression estimates of the percentage change in laboratory values at 15 days post admission (after this point, only a minority of patients were still hospitalised) was correlated across the hospitals: for both the discharged and expired groups, these correlations were significant and positive (discharged:

$\rho=0.94, t_{17}=11.25, p<0.01$; expired: $\left.\rho=0.94, t_{17}=11.58, p<0.01\right)$. 

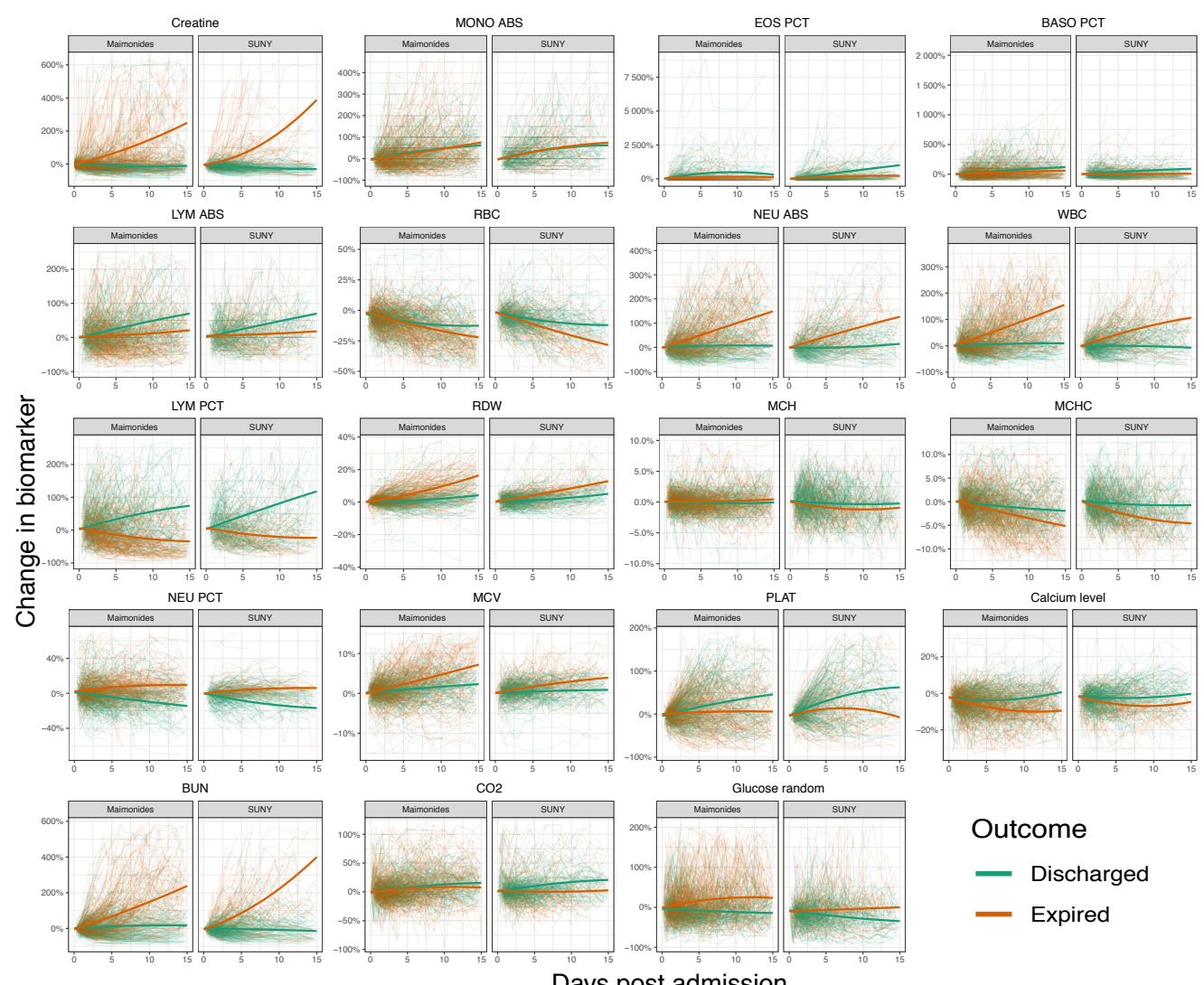

Outcome

- Discharged

- Expired

Fig 3. Comparing time trends in laboratory values across hospitals. The horizontal axis shows the days post admission and the vertical axis shows the percentage change in biomarker values from their initial values. Each panel displays trends for an individual biomarker; within the subpanels of each of these, we show the results for each of the hospitals. Individual graphs show the dynamics of the individual patients' laboratory values (thin coloured lines) and the time trends (thick coloured lines) estimated assuming a quadratic regression function. Line colouring indicates the outcome of an individual patient (thin lines) or overall group being considered for regressions (thick lines). Note that for plotting we only display data up until day 15 post admission, since, after this point, there were relatively few patients still hospitalised.

\section{Quantifying risk associated with dynamic biomarker measurements}

In what follows, we compare the the univariate ORs associated with daily mortality risk for each of the common biomarkers across the two hospitals as derived from the Markov model (described in Methods). There was a strong positive correlation $\left(\rho=0.85, t_{17}=6.52, p<0.01\right)$ in the estimates of the ORs between the hospitals (Fig 4 ). For 16/19 of the variables, the ORs estimates agreed in their "sign": 9/19 of the variables had an OR $>1$ (posterior medians) in both analyses; 7/19 had an OR $<1$ in both analyses. For the remaining variables, the ORs from Maimonides tended to be lower than those of SUNY.

Based on these estimates, increases in MCV, decreases in LYM PCT, and decreases in $\mathrm{CO}_{2}$ throughout a patient's stay were associated with the strongest increase in mortality risk.

Next, we compare the ORs estimated from the Markov model with the full set of 


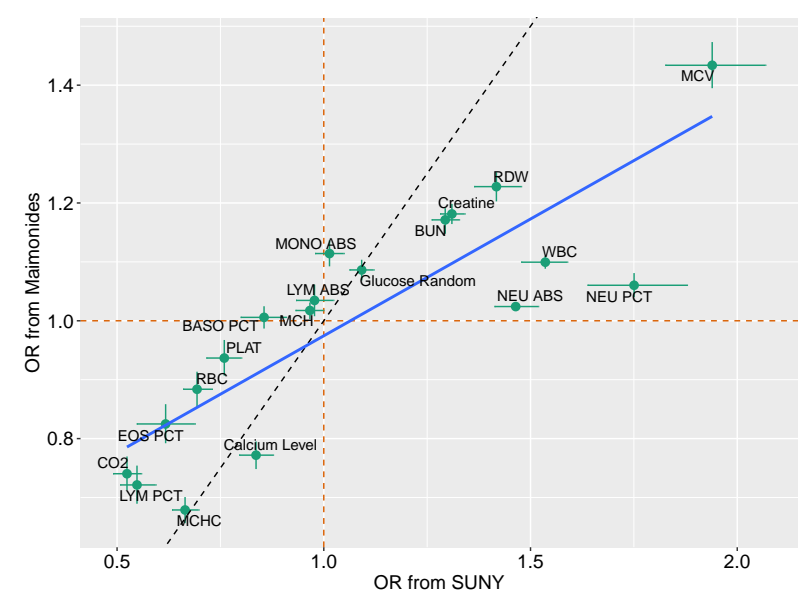

Fig 4. Comparing univariate ORs for dynamic laboratory measurements. The horizontal axes displays the ORs for daily mortality risk from SUNY and the vertical axes show the ORs from Maimonides. Points show the posterior median ORs; the whiskers display the $25 \%$ and $75 \%$ posterior quantiles. The orange dashed lines show the OR $=1$ cases; the dashed black lines indicate equality across ORs calculated across the hospitals. The blue line shows least squares regression lines using the posterior median ORs.

regressors (the "post-admission" set described in Methods): in doing so, we aim to isolate those factors which convey the most independent predictive power. In Fig S7A, we correlate the ORs associated with the dynamic biomarker measurements across both hospitals. In contrast to the univariate estimates, the relationship between the estimates was less clear and the correlation in estimates was insignificant

$\left(\rho=0.06, t_{17}=0.23, p>0.05\right)$. This result was, however, largely driven by the estimates for MCHC and MCV which were very different across the hospitals; when the estimates were replotted with these removed (Fig S7B), the estimates appeared positively correlated.

\section{Assessing generalisation of mortality predictions from the Markov model}

Across the different regressor sets, predictive accuracy was consistent across the hospitals (Fig 5A). In all cases, the posterior median predictive accuracy using data from Maimonides resulted in slightly higher prediction than when using data from SUNY: likely due to the higher sample size for Maimonides. The results also show the predictive power of dynamic laboratory measurements (included in the "post-admission" set), which resulted in a substantial boost in accuracy across both hospitals over a model including only those available at admission ("admission").

We next used the model using all available post-admission variables to probe its predictive performance for those groups patients who went on to be discharged and died. To do so, we pooled predictions across both independent hospital testing sets. The resultant confusion matrix is shown in Fig $5 \mathrm{~B}$. This indicates that the model had a higher sensitivity to determine patients that would eventually be discharged (posterior mean: $79.2 \%$ ) compared to those who would go on to die (61.0\%).

We next assessed the reduction in accuracy when predicting patient outcomes in the same hospital versus a different hospital. We did this by using validation sets either comprised of separate data from the same hospital ("within') or a different hospital 
("between"). In Fig S8, we show the predictive accuracy for models fitted using data from SUNY (left panel) and Maimonides (right panel). Point colour indicates whether the validation set comprised patients from within the same hospital (green) or a different hospital (orange). Note that, in this analysis, the requirement for an independent within-hospital datasets to fit the model meant that the training datasets were smaller than those used to produce Fig 5A: resulting in slightly lower overall accuracy. In almost all cases, median predicted accuracy when predicting outcomes within the same hospital was higher than that when predicting those in a different hospital. The difference, however, was relatively small (mean difference in posterior medians: $2.1 \%$ ), indicating that the predictions generalised well from one location to another.
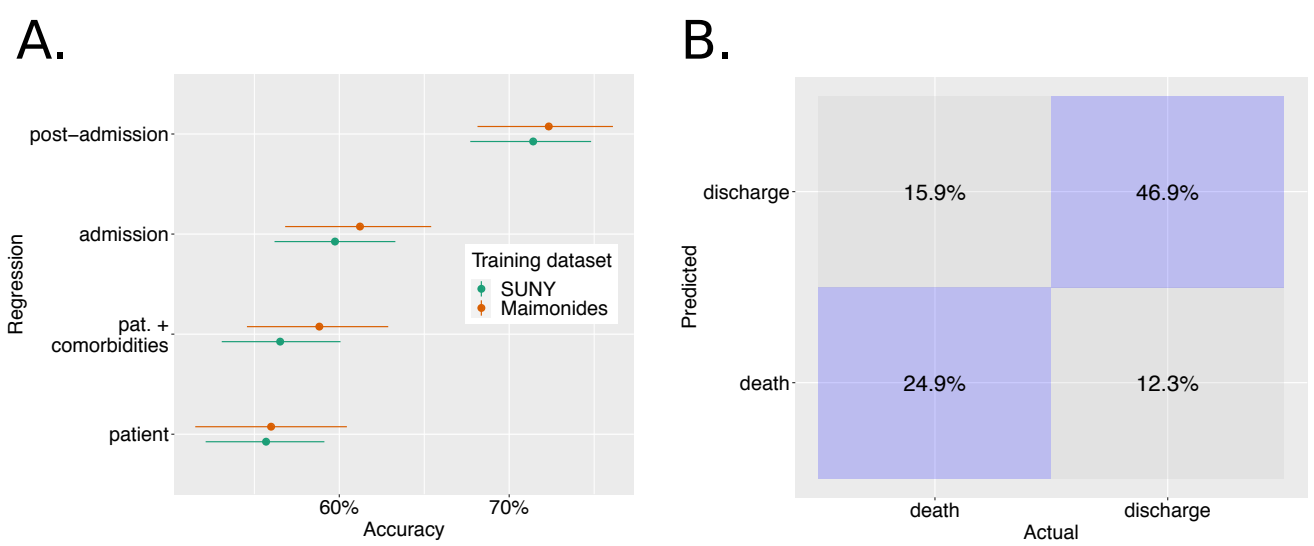

Fig 5. Predictive accuracy in patient outcomes for held-out hospital. Panel A shows the accuracy in predicting outcomes across four regression sets: Colours indicate the hospital whose data was used to train the model: so, for example, "SUNY" indicates that data from this hospital was used to fit the model which was then tested on Maimonides. The horizontal axis shows the accuracy in predicting patient outcomes (i.e. death or discharge) using a Markov regression model with covariate sets as named on the vertical axis. The points and whiskers indicate the posterior medians and 2.5\%-97.5\% posterior intervals for the percentage of patients whose outcome was correctly determined across posterior draws. Panel B shows a confusion matrix for between-hospital prediction using Markov model with the post-admission covariate set. Here, the values show the mean percentage of each outcome type correctly predicted across all posterior samples.

To determine whether there were subgroups of patients where the model performed better or worse, we examined the factors that influenced the predictive accuracy for those patients who went on to die in each of the hospitals. To do so, we used the models that were trained on all the data from one hospital (i.e. those used to produce Fig 5 A). We then used a random forest to predict posterior median predictive accuracy for each of the patients, as a function of their time-invariant characteristics. Then using the "impurity" measure of variable importance, we identified those variables that were associated with differences in predictive accuracy. The top three factors were: the time since the first patient was admitted to that hospital with COVID-19, whether the patient had a history of coronary artery disease and whether their self-reported ethnicity was categorised as non-Caucasian. These three variables were then included in a linear regression to predict median predictive accuracy. This regression indicated that having a history of coronary artery disease led to improved predictive power $\left(\beta=0.14, t_{478}=6.52, p<0.01\right) ;$ other factors were insignificant. 


\section{Discussion}

A number of studies have demonstrated that dynamic changes in certain laboratory tests may have potential as COVID-19 prognostic factors [12,14,15. Here, we demonstrate the external validation of a number of these dynamic biomarkers. In accordance with existing studies, we find a number of biomarkers at presentation (or when measured at a single time-point) increased mortality risk across both hospital cohorts (univariate ORs): these included eosinopenia [24], thrombocytopenia 25, 26], lymphocytopenia 26, 27] and increased blood urea concentration (in our case indicated by BUN) 11]. In addition, we quantified the reduction in mortality risk associated with changes in these biomarkers. Interestingly, we identified a number of biomarkers that have little prognostic value at presentation whereas their dynamic changes do: increases in MCV and decreases in MCHC post-admission, for example, increased mortality risk according the univariate ORs. The external validation of the multivariate ORs for these variables indicated, however, these findings were inconsistent across hospitals. These results highlight the potential importance of measuring dynamic changes in biomarkers for patient prognosis and the importance of external validation. Our model could better predict outcomes for patients who went on to be discharged opposed to those who eventually died, indicating the challenges in assessing mortality risk in hospitalised COVID-19 patients.

Our study suffered from a number of limitations. Data availability limited the prognostic factors tested, and future work is therefore required to quantify the mortality risk associated with dynamic changes in other prognostic factors that are known to be important at presentation. These include abnormal biomarkers of inflammation, myocardial injury, acute respiratory distress syndrome (ARDS) and coagulopathy 25, 27. We also did not include time-dependent changes in certain chemokines and cytokines, which can also indicate disease progression 12,28 . Additionally, we did not account for the potential impact of patient treatment on dynamic changes in biomarkers or on outcomes. Mechanical ventilation of patients with ARDS, for example, is used to maintain certain arterial $\mathrm{pCO}_{2}$ values, and both mechanical ventilation and certain COVID-19 pharmaceutical treatments can influence inflammatory markers 29,30. We considered patients solely hospitalised during early to mid-2020 within a single region (New York), but novel variants and existing immunity may alter survival 31. Within certain settings, patient survival has improved throughout the course of the pandemic [32, and temporal recalibration of multivariate regression models, which aim to quantify the OR of survival for different prognostic factors, is therefore necessary to ensure survival is not under- or overestimated [33].

Whilst our model performed well across the two cohorts examined, we caution against its use as a dynamic prognostic model in clinical settings. In order for it to be used as thus, any such model requires training and evaluation over a much larger sample size across multiple settings including the full set of factors implicated with risk. The ease of use in a clinical setting and effects on clinicians' behaviour, comparison with existing prognostic models, cost-effectiveness and impact on patient health must also be assessed prior to the implementation of any prognostic model 34 .

\section{References}

1. Supady A, Curtis JR, Abrams D, Lorusso R, Bein T, Boldt J, et al. Allocating scarce intensive care resources during the COVID-19 pandemic: practical challenges to theoretical frameworks. The Lancet Respiratory Medicine. 2021;9(4):430-434. 
2. Wynants L, Van Calster B, Collins GS, Riley RD, Heinze G, Schuit E, et al. Prediction models for diagnosis and prognosis of COVID-19: systematic review and critical appraisal. BMJ. 2020;369.

3. Upshaw TL, Brown C, Smith R, Perri M, Ziegler C, Pinto AD. Social determinants of COVID-19 incidence and outcomes: A rapid review. PLOS ONE. $2021 ; 16(3): 1-22$.

4. Williamson EJ, Walker AJ, Bhaskaran K, Bacon S, Bates C, Morton CE, et al. Factors associated with COVID-19-related death using OpenSAFELY. Nature. 2020;584(7821):430-436.

5. Mena GE, Martinez PP, Mahmud AS, Marquet PA, Buckee CO, Santillana M. Socioeconomic status determines COVID-19 incidence and related mortality in Santiago, Chile. Science. 2021;.

6. Li SL, Pereira RHM, Prete Jr CA, Zarebski AE, Emanuel L, Alves PJH, et al. Higher risk of death from COVID-19 in low-income and non-white populations of São Paulo, Brazil. BMJ Global Health. 2021;6(4).

7. Navaratnam AV, Gray WK, Day J, Wendon J, Briggs TWR. Patient factors and temporal trends associated with COVID-19 in-hospital mortality in England: an observational study using administrative data. The Lancet Respiratory Medicine. 2021;9(4):397-406.

8. Altman DG, Vergouwe Y, Royston P, Moons KGM. Prognosis and prognostic research: validating a prognostic model. BMJ. 2009;338.

9. Gupta RK, Marks M, Samuels THA, Luintel A, Rampling T, Chowdhury H, et al. Systematic evaluation and external validation of 22 prognostic models among hospitalised adults with COVID-19: an observational cohort study. European Respiratory Journal. 2020;56(6).

10. Gupta RK, Harrison EM, Ho A, Docherty AB, Knight SR, van Smeden M, et al. Development and validation of the ISARIC $4 \mathrm{C}$ deterioration model for adults hospitalised with COVID-19: a prospective cohort study. The Lancet Respiratory Medicine. 2021;9(4):349-359.

11. Knight SR, Ho A, Pius R, Buchan I, Carson G, Drake TM, et al. Risk stratification of patients admitted to hospital with covid-19 using the ISARIC WHO Clinical Characterisation Protocol: development and validation of the $4 \mathrm{C}$ Mortality Score. BMJ. 2020;370.

12. Abers MS, Delmonte OM, Ricotta EE, Fintzi J, Fink DL, de Jesus AAA, et al. An immune-based biomarker signature is associated with mortality in COVID-19 patients. JCI Insight. 2021;6(1).

13. Chen R, Sang L, Jiang M, Yang Z, Jia N, Fu W, et al. Longitudinal hematologic and immunologic variations associated with the progression of COVID-19 patients in China. Journal of Allergy and Clinical Immunology. 2020;146(1):89-100.

14. Berzuini C, Hannan C, King A, Vail A, O'Leary C, Brough D, et al. Value of dynamic clinical and biomarker data for mortality risk prediction in COVID-19: a multicentre retrospective cohort study. BMJ Open. 2020;10(9).

15. Momeni-Boroujeni A, Mendoza R, Stopard IJ, Lambert B, Zuretti A. A Dynamic Bayesian Model for Identifying High-Mortality Risk in Hospitalized COVID-19 Patients. Infectious Disease Reports. 2021;13(1):239-250. 
16. Samprathi M, Jayashree M. Biomarkers in COVID-19: An Up-To-Date Review. Frontiers in Pediatrics. 2021;8:972.

17. Bates D, Sarkar D, Bates MD, Matrix L. The lme4 package. R package version. $2007 ; 2(1): 74$.

18. Collett D. Modelling survival data in medical research. CRC press; 2015.

19. Wolbers M, Koller MT, Witteman JC, Steyerberg EW. Prognostic models with competing risks: methods and application to coronary risk prediction. Epidemiology. 2009; p. 555-561.

20. Betancourt M. A conceptual introduction to Hamiltonian Monte Carlo. arXiv preprint arXiv:170102434. 2017;.

21. Carpenter B, Gelman A, Hoffman MD, Lee D, Goodrich B, Betancourt M, et al. Stan: a probabilistic programming language. Grantee Submission. 2017;76(1):1-32.

22. Gelman A, Carlin JB, Stern HS, Dunson DB, Vehtari A, Rubin DB. Bayesian data analysis. Chapman Hall, London; 2013.

23. Lambert B. A student's guide to Bayesian statistics. Sage; 2018.

24. Xie G, Ding F, Han L, Yin D, Lu H, Zhang M. The role of peripheral blood eosinophil counts in COVID-19 patients. Allergy. 2021;76(2):471-482.

25. Perez-Guzman PN, Daunt A, Mukherjee S, Crook P, Forlano R, Kont MD, et al. Clinical Characteristics and Predictors of Outcomes of Hospitalized Patients With Coronavirus Disease 2019 in a Multiethnic London National Health Service Trust: A Retrospective Cohort Study. Clinical Infectious Diseases. 2020;.

26. Malik P, Patel U, Mehta D, Patel N, Kelkar R, Akrmah M, et al. Biomarkers and outcomes of COVID-19 hospitalisations: systematic review and meta-analysis. BMJ Evidence-Based Medicine. 2021;26(3):107-108.

27. Paranjpe I, Russak AJ, De Freitas JK, Lala A, Miotto R, Vaid A, et al. Retrospective cohort study of clinical characteristics of 2199 hospitalised patients with COVID-19 in New York City. BMJ Open. 2020;10(11).

28. Laing AG, Lorenc A, del Molino del Barrio I, Das A, Fish M, Monin L, et al. A dynamic COVID-19 immune signature includes associations with poor prognosis. Nature Medicine. 2020;26(10):1623-1635.

29. Ranieri VM, Suter PM, Tortorella C, De Tullio R, Dayer JM, Brienza A, et al. Effect of Mechanical Ventilation on Inflammatory Mediators in Patients With Acute Respiratory Distress Syndrome: a Randomized Controlled Trial. JAMA. $1999 ; 282(1): 54-61$.

30. Del Valle DM, Kim-Schulze S, Huang HH, Beckmann ND, Nirenberg S, Wang B, et al. An inflammatory cytokine signature predicts COVID-19 severity and survival. Nature medicine. 2020;26(10):1636-1643.

31. Challen R, Brooks-Pollock E, Read JM, Dyson L, Tsaneva-Atanasova K, Danon L. Risk of mortality in patients infected with SARS-CoV-2 variant of concern 202012/1: matched cohort study. BMJ. 2021;372. 
32. Dennis JM, McGovern AP, Vollmer SJ, Mateen BA. Improving Survival of Critical Care Patients With Coronavirus Disease 2019 in England: A National Cohort Study, March to June 2020*. Critical Care Medicine. 2021;49(2).

33. Booth S, Riley RD, Ensor J, Lambert PC, Rutherford MJ. Temporal recalibration for improving prognostic model development and risk predictions in settings where survival is improving over time. International Journal of Epidemiology. 2020;49(4):1316-1325.

34. Steyerberg EW, Moons KGM, van der Windt DA, Hayden JA, Perel P, Schroter S, et al. Prognosis Research Strategy (PROGRESS) 3: Prognostic Model Research. PLOS Medicine. 2013;10(2):1-9.

\section{Acknowledgements}

The authors wish to thank the study participants for agreeing to the use of their data. 